PROCEEDINGS OF THE

AMERICAN MATHEMATICAL SOCIETY

Volume 131, Number 3, Pages 937-939

S 0002-9939(02)06583-8

Article electronically published on June 13, 2002

\title{
APPROXIMATION OF MEASURABLE MAPPINGS BY SEQUENCES OF CONTINUOUS FUNCTIONS
}

\author{
SURJIT SINGH KHURANA
}

(Communicated by Claudia M. Neuhauser)

\begin{abstract}
Let $X$ be a completely regular Hausdorff space, $\mu$ a positive, finite Baire measure on $X$, and $E$ a separable metrizable locally convex space. Suppose $f: X \rightarrow E$ is a measurable mapping. Then there exists a sequence of functions in $C_{b}(X) \otimes E$ which converges to $f$ a.e. [ $\mu$ ]. If the function $f$ is assumed to be weakly continuous and the measure $\mu$ is assumed to be $\tau$-smooth, then a separability condition is not needed.
\end{abstract}

\section{InTRODUCTION AND NOTATIONS}

In [9], it is proved that for a metric space $X$ with a Borel measure $\mu$, a measurable mapping $f: X \rightarrow E$ is almost everywhere the limit of a sequence of continuous functions if $E$ is a separable Banach space; this is an extension of an earlier result in [8]. Though the result is stated to be extended to a locally convex space $E$ in [4] (Theorem 1, p. 1513), what is proved in that theorem (4], Theorem 1) gives a sequence of continuous functions only when $E$ is metrizable.

In this paper we give some extensions of this theorem by assuming $X$ to be completely regular and $E$ a separable metrizable locally convex space. If we assume the function $f$ to be weakly continuous and the measure $\mu$ to be $\tau$-smooth, then the separability condition on $E$ is not needed. Our proofs are very different and the results follow easily from the regularity of measures.

For topological measure theory, notations and results from [7] and [10] will be used. All vector spaces are taken over $K$, the field of real or complex numbers (called scalars). For a completely regular Hausdorff space $X, C_{b}(X)$ will denote all bounded continuous on $X$. For locally convex spaces, notations and results of [6] will be used. For a locally convex space $E, E^{\prime}$ will denote its dual. For a completely regular Hausdorff space $\mathrm{X}$ and a locally convex space $E, C_{b}(X, E)$ will denote all bounded and continuous $E$-valued functions on $X$, and $C_{b}(X) \otimes E$ will denote the tensor product of $C_{b}(X)$ and $E$.

If, for $i=1,2, X_{i}$ are sets and $\mathcal{A}_{i}$ are sigma-algebras on $X_{i}$, then a mapping $\rho: X_{1} \rightarrow X_{2}$ is called measurable if $\rho^{-1}(B) \in \mathcal{A}_{1}$ for every $B \in \mathcal{A}_{2}$. If $X_{2}$ is a metrizable locally convex space, then the sigma-algebra $\mathcal{A}_{2}$ will always be the class of all Borel subsets of $X_{2}$. For measure theory we refer to [1]. If $(X, \mathcal{A}, \mu)$ is a finite measure space and $E$ a metrizable locally convex space, a mapping $f: X \rightarrow E$

Received by the editors August 18, 2001 and, in revised form, October 10, 2001.

2000 Mathematics Subject Classification. Primary 60B05, 28C15; Secondary 60B11, 28 B05.

Key words and phrases. Baire measures, $\tau$-smooth measures, support of a measure. 
will be called strongly measurable if there exists a sequence of simple measurable functions, converging to $f$ a.e. $[\mu]$ ([1], Theorem 2, p. 99).

\section{MAin Results}

Lemma 1. Let $X$ be a completely regular Hausdorff space and $\mu$ a positive, finite Baire measure on $X$ and $E$ a separable normed space. Suppose $f: X \rightarrow E$ is a measurable mapping. Then there exists a sequence of functions in $C_{b}(X) \otimes E$ which converges to $f$ a.e. $[\mu]$.

Proof. For every $g \in E^{\prime}, g \circ f$ is measurable. Since $E$ is separable, this implies that $f$ is strongly measurable (1], Proposition 12, p. 93). Thus there exists a sequence $\left\{h_{n}\right\}$ of simple measurable functions such that $h_{n} \rightarrow f$ a.e. $[\mu]$. By regularity of Baire measures ([7], [10]), for a Baire subset $B \subset X$ and $c>0$, there is a $\phi \in C_{b}(X)$ such that $\int\left|\chi_{B}-\phi\right| d \mu<c$. This means, for each $h_{n}$, there is $\overline{h_{n}} \in C_{b}(X) \otimes E$ such that $\int\left\|h_{n}^{-}-h_{n}\right\| d \mu<\frac{1}{2^{n}}$. Thus there exists a subsequence of $\left\{h_{n}^{-}-h_{n}\right\}$ which converges to 0 , a.e. $(\mu)$. Thus $\overline{h_{n}} \rightarrow f$ a.e. $(\mu)$. This proves the results.

Now we prove the following theorem for separable metrizable locally convex spaces.

Theorem 2. Let $X$ be a completely regular Hausdorff space and $\mu$ a positive, finite Baire measure on $X$ and $E$ a separable metrizable locally convex space. Suppose $f: X \rightarrow E$ is a measurable mapping. Then there exists a sequence of functions in $C_{b}(X) \otimes E$ which converges to $f$ a.e. $[\mu]$.

Proof. Let $\|\cdot\|_{n}$ be an increasing family of semi-norms generating the topology of $E$. By Lemma 1 and Egoroff's theorem ([1, Theorem 1, p. 94), there exists, for every $k$, a Baire set $A_{k}$ with $\mu\left(A_{k}\right)<\frac{1}{2^{k}}$, and a $g_{k} \in C_{b}(X) \otimes E$ with $\left\|g_{k}-f\right\|_{k}<\frac{1}{2^{k}}$ on $X \backslash A_{k}$. From this it follows that $g_{k} \rightarrow f$ on $X \backslash A$, where $A=\bigcap_{n=1}^{\infty} \bigcup_{i=n}^{\infty} A_{i}$. Since $\mu(A)=0$, the result follows.

In the next theorem, we remove the condition of separability but put some additional conditions on the function $f$ and the measure $\mu$.

Theorem 3. Let $X$ be a completely regular Hausdorff space and $\mu$ a positive, finite $\tau$-smooth measure on $X$ and $E$ a metrizable locally convex space. Suppose $f: X \rightarrow$ $E$ is a weakly continuous mapping. Then there exists a sequence of functions in $C_{b}(X) \otimes E$ which converges to $f$ a.e. $[\mu]$.

Proof. Consider the measure $\lambda=\mu \circ f^{-1}$ on Borel subsets of $E$ with weak topology. This measure is $\tau$-smooth and so has a support $Y$, a weakly closed subset of $E$. Let $X_{0}=f^{-1}(Y) . \quad X_{0}$ is a closed subset of $X$ and $\mu\left(X_{0}\right)=\mu(X)$. Suppose, for some $g \in E^{\prime}, g \circ f=0$ a.e. $[\mu]$. We claim that $g \circ f=0$ on $X_{0}$. To prove this, let $V=\{t \in Y:|g(t)|>0\}$ and $A=\left\{x \in X_{0}:|g \circ f(x)|>0\right\}$. It is easily verified that $A=f^{-1}(V)$. If $V$ is non-void, then, being open in $Y, \lambda(V)>0$ and so $\mu(A)>0$. But this is a contradiction since $g \circ f=0$ a.e. $[\mu]$. Thus $A$ is a void set and so the claim is established. By [5] (Theorem 6, p. 813, see also [2], [3]), $f$ is strongly meaurable. Since $\mu$ is $\tau$-smooth, for any Borel subset $B \subset X$ and $c>0$, there is a $\phi \in C_{b}(X)$ such that $\int\left|\chi_{B}-\phi\right| d \mu<c([7],[10])$; this is exactly the main result used in Lemma 1 for Baire measure $\mu$ and Baire subset $B \subset X$. Proceeding exactly as in Lemma 1 and Therorem 2, the result follows. 


\section{REFERENCES}

[1] Dinculeanu, N., Vector measures, Pergamon Press, 1967. MR 34:6011b

[2] Ionescu, Tulcea, A, On pointwise convergence, compactness and equicontinuity in the lifting topology. I, Z. Wahrscheinlichkeitstheorie und Verw. Gebiete 26(1973), 197-205. MR 53:8898a

[3] Ionescu, Tulcea, A, On pointwise convergence, compactness and equicontinuity in the lifting topology. II, Advances in Math. 12(1974), 171-177. MR 53:8898b

[4] Kawabe, Jun, The structure of measurable mappings with values in locally convex spaces, Proc. Amer. Math. Soc. 124(1996), 1513-1515. MR 96g:28003

[5] Khurana, S. S., Strong measurability in Frechet spaces, Indian J. Pure Applied Math. 10(1979), 810-814. MR 80j:28015

[6] Schaeffer, H. H., Topological Vector spaces, Springer Verlag, 1986.

[7] Wheeler, R. F., Survey of Baire measures and strict topologies, Expos. Math. 1(1983), 97-190. MR 85b:46035

[8] Wisniewski, Andrzej, The structure of measurable mappings on metric spaces, Proc. Amer. Math. Soc. 122(1994), 147-150. MR 94k:28006

[9] Wisniewski, Andrzej, The continuous approximation of measurable mappings, Demonstratio Math. 29(1996), 529-531. MR 98c:28003

[10] Varadarajan, V. S., Measures on topological spaces, Amer. Math. Soc. Transl. (2)48(1965), 161-220. MR 26:6342

Department of Mathematics, University of Iowa, Iowa City, Iowa 52242

E-mail address: khurana@math.uiowa.edu 